\title{
Black Hole Radiation (with and) without Weyl Anomaly!
}

\author{
G. Amelino-Cameliaf and D. Seminara \\ Center for Theoretical Physics \\ Laboratory for Nuclear Science and Department of Physics \\ Building 6, Massachusetts Institute of Technology \\ Cambridge, Massachusetts 02139, U.S.A.
}

\begin{abstract}
In the semiclassical analysis of black hole radiation in matter-coupled dilaton gravity, a one-parameter " $k$ "-family of measures for the path integral quantization of the matter fields is considered. The Weyl anomaly is proportional to the parameter $k$, but the black hole radiation seen by minkowskian observers at future null infinity is $k$-independent.
\end{abstract}

Submitted to: Classical and Quantum Gravity

MIT-CTP-2443

\footnotetext{
${ }^{1}$ This work is supported in part by funds provided by the U.S. Department of Energy (D.O.E.) under cooperative agreement \#DE-FC02-94ER40818, and by Istituto Nazionale di Fisica Nucleare (INFN, Frascati, Italy).

${ }^{2}$ Present address: Theoretical Physics, Oxford University, 1 Keble Rd., Oxford OX1 3NP, U.K.
} 


\section{Introduction}

In the conventional semiclassical analysis [1] of black hole radiation [2] in matter-coupled dilaton gravity (MCDG)

$$
S_{D}=\int d^{2} x \sqrt{-g} e^{-2 \phi}\left[R+4(\nabla \phi)^{2}+4 \lambda^{2}\right]-\frac{1}{2} \sum_{n=1}^{N} \int d^{2} x \sqrt{-g} g^{\mu \nu} \partial_{\mu} f_{n} \partial_{\nu} f_{n},
$$

where $g, \phi$, and $f_{n}$ are the metric, dilaton, and matter fields respectively, a central role [1, 3] is played by the Weyl anomaly. One starts by treating classically the gravitational collapse, and then the matter degrees of freedom are quantized in the background of the resulting black hole metric. The Weyl anomaly

$$
g^{\mu \nu} T_{\mu \nu}=\frac{N}{24 \pi} R(g)
$$

where $T_{\mu \nu}$ is the matter energy-momentum tensor [4]

$$
T_{\mu \nu} \equiv \sum_{n=1}^{N}<\partial_{\mu} f_{n} \partial_{\nu} f_{n}-\frac{1}{2} g_{\mu \nu} g^{\alpha \beta} \partial_{\alpha} f_{n} \partial_{\beta} f_{n}>_{g}
$$

is a consequence of the diffeomorphism-invariant quantization of the matter fields; for example, in the path integral formulation, Eq.(2) follows from choosing the diffeomorphisminvariant measure [5]

$$
\int \mathcal{D} \delta f_{n} \exp \left(i \int d^{2} x \sqrt{-g} \delta f_{n} \delta f_{n}\right)=1
$$

which is not Weyl-invariant.

Once appropriate physical boudary conditions are imposed, Eq.(2) and the covariant conservation of the matter energy-momentum tensor

$$
\nabla_{\mu}\left(g^{\mu \nu} T_{\nu \alpha}\right)=0
$$

determine $T_{\mu \nu}$, which has three independent components in $1+1$ dimensions, and therefore determine the black hole radiation.

The Weyl anomaly is usually considered[1, 3] to be a crucial ingredient of black hole radiation because for a traceless matter energy-momentum tensor Eq.(5) would lead to no 
radiation. In order to achieve a deeper understanding of the relation between Weyl anomaly and black hole radiation, in this Letter we study how the conventional analysis of black hole radiation in MCDG is affected by the modifications to Eqs.(2) and (5) that arise in the alternative approaches to the quantization of the matter fields which have been recently considered in Refs.[5-9].

\section{$2 \quad k$-dependent Anomaly Relations}

In the path integral formulation, the alternative approaches to the quantization of the matter fields considered in Refs. [6, 7] correspond to the choice of measure

$$
\int \mathcal{D} \delta f_{n} \exp \left(i \int d^{2} x(-g)^{\frac{k}{2}} \delta f_{n} \delta f_{n}\right)=1
$$

where $k$ is a fixed real parameter.

We observe that this choice of measure is invariant under infinitesimal variations of the form[

$$
\delta x^{\mu}=v^{\mu}, \quad \delta g_{\mu \nu}=v^{\alpha} \partial_{\alpha} g_{\mu \nu}+g_{\alpha \nu} \partial_{\mu} v^{\alpha}+g_{\alpha \mu} \partial_{\nu} v^{\alpha}+\frac{1-k}{k} g_{\mu \nu} \partial_{\alpha} v^{\alpha}
$$

In order to render this formula valid for all $k$ 's, we prescribe that the singular limit $k \rightarrow 0$, which corresponds to the theory considered in Refs. [7-9], be formally taken so that $\partial_{\mu} v^{\mu} \rightarrow k w$ for $k \rightarrow 0$, where $w$ is an arbitrary function. Following this limiting procedure, at $k=0$ the variations (7) take the form

$$
\delta x^{\mu}=v^{\mu}, \quad \delta g_{\mu \nu}=v^{\alpha} \partial_{\alpha} g_{\mu \nu}+g_{\alpha \nu} \partial_{\mu} v^{\alpha}+g_{\alpha \mu} \partial_{\nu} v^{\alpha}+w g_{\mu \nu}, \quad \text { with } \partial_{\mu} v^{\mu}=0
$$

which indeed reproduce the invariance[7-9] of the measure (6), at $k=0$, under diffeomorphisms of unit Jacobian $\left(\partial_{\mu} v^{\mu}=0\right)$ and Weyl transformations.

Note that (7) and (8) indicate that diffeomorphisms of unit Jacobian are a symmetry of the measure (6) for every value of $k$.

\footnotetext{
${ }^{3}$ Note that the matter action in (1) is invariant under diffeomorphisms and Weyl transformations [and therefore in particular is invariant under the transformations (7)]; this is related to the fact that, if $g_{\mu \nu}$ is a density of weight $\rho$, the transformations of $\sqrt{-g} g^{\mu \nu}$ under diffeomorphisms are independent of $\rho$.
} 
As a result of the properties of the measure (6), the matter energy-momentum tensor satisfies the following anomaly relations

$$
\begin{aligned}
g^{\mu \nu} T_{\mu \nu} & =k \frac{N}{24 \pi} g^{\frac{k-1}{2}} R(\hat{g}), \\
\nabla_{\mu}\left(g^{\mu \nu} T_{\nu \alpha}\right) & =(k-1) \frac{N}{48 \pi} \frac{1}{\sqrt{-g}} \partial_{\alpha}\left[g^{\frac{k}{2}} R(\hat{g})\right],
\end{aligned}
$$

where $\hat{g}_{\mu \nu} \equiv(-g)^{\frac{k-1}{2}} g_{\mu \nu}$. Obviously for $k=1$ Eqs.(9) and (10) reproduce Eqs.(2) and (5), and for $k=0$ they reproduce the corresponding relations encountered in Refs. 8, 9, 10]. For our investigation of the relation between Weyl anomaly and black hole radiation, it is especially important that (9) is directly proportional to $k$, which, in particular, implies that there is no Weyl anomaly in the $k=0$ limit.

The invariance under the transformations (17) is encoded in the fact that

$$
\sqrt{-g} \nabla_{\mu}\left(g^{\mu \nu} T_{\nu \alpha}\right)+\frac{1-k}{2 k} \partial_{\alpha}\left[\sqrt{-g} g^{\mu \nu} T_{\mu \nu}\right]=0
$$

which is consistent with (9) and (10).

Interestingly, the relations (9), (10), and (11) can all be rewritten rather elegantly in terms of $\hat{g}$ and $\hat{\nabla}$, the covariant derivative computed with the metric $\hat{g}$,

$$
\begin{aligned}
\hat{g}^{\mu \nu} T_{\mu \nu} & =k \frac{N}{24 \pi} R(\hat{g}), \\
\hat{\nabla}_{\mu}\left(\hat{g}^{\mu \nu} T_{\nu \alpha}\right) & =(k-1) \frac{N}{48 \pi} \partial_{\alpha} R(\hat{g}), \\
\hat{\nabla}_{\mu}\left(\hat{g}^{\mu \nu} T_{\nu \alpha}\right) & +\frac{1-k}{2 k} \partial_{\alpha}\left(\hat{g}^{\mu \nu} T_{\mu \nu}\right)=0 .
\end{aligned}
$$

This is a consequence 11] of the fact that the transformations (7) can be obtained as the realization of the diffeomorphism group on a tensorial density of weight $\frac{1-k}{k}$. (N.B.: $g_{\mu \nu}$ has weight $\frac{1-k}{k}$ with respect to the metric $\hat{g}_{\mu \nu}$.)

The fact that the right-hand sides of Eqs.(9) and (10) [or equivalently (12) and (13)] do not transform covariantly under general diffeomorphisms implies that $T_{\mu \nu}$ is not a tensor. One can show that, under a coordinate redefinition $x^{\mu} \rightarrow y^{\mu}, T_{\mu \nu}$ transforms as follows

$$
T_{\mu \nu}^{(x)} \rightarrow T_{\mu \nu}^{(y)}=\left(T_{\alpha \beta}^{(x)}+\Delta_{\alpha \beta}^{(x, y)}\right) \frac{d x^{\alpha}}{d y^{\mu}} \frac{d x^{\beta}}{d y^{\nu}}
$$


where

$$
\begin{aligned}
\Delta_{\alpha \beta}^{(x, y)}= & \frac{N(1-k)}{24 \pi}\left(\nabla_{\alpha} \nabla_{\beta} \ln J-g_{\alpha \beta} \square \ln J\right)+\frac{N(1-k)^{2}}{96 \pi}\left(g_{\alpha \beta} \nabla_{\gamma} \ln J \nabla^{\gamma} \ln J-2 \nabla_{\alpha} \ln J \nabla_{\beta} \ln J\right. \\
& \left.+\nabla_{\alpha} \ln \sqrt{-g} \nabla_{\beta} \ln J+\nabla_{\beta} \ln \sqrt{-g} \nabla_{\alpha} \ln J-g_{\alpha \beta} \nabla^{\gamma} \ln \sqrt{-g} \nabla_{\gamma} \ln J+g_{\alpha \beta} \square \ln J\right)
\end{aligned}
$$

and $J$ is the Jacobian of the transformation $x^{\mu} \rightarrow y^{\mu}$.

Notice that $\Delta_{\alpha \beta}^{(x, y)}=0$ whenever $J$ is constant. This implies that $T_{\mu \nu}$ transforms covariantly not only under diffeomorphisms of unit Jacobian, but also under dilatations $\left(y^{\mu}=c x^{\mu}\right.$ with constant $c$ ); in fact, a general coordinate redefinition of constant Jacobian can be obtained as the composition of a diffeomorphism of unit Jacobian and a dilatation. The covariance of $T_{\mu \nu}$ under this diffeomorphism subgroup which is larger than the one leaving invariant the measure (6) can be understood[11] as a consequence of the fact that in $1+1$ dimensions $\sqrt{-g} R(g)$ is a total derivative [9].

\section{Black Hole Radiation}

\subsection{Conformal-Gauge Analysis for $k=1$}

We now turn to the study of black hole radiation, starting with a brief review of the conventional $(k=1)$ approach to the problem, i.e. assuming that the relations (2) and (5) hold. For simplicity, we limit our analysis to the example of black hole discussed in Ref. [1], and keep our notation consistent with the one of Ref.[1]]; in particular, we introduce light-cone coordinates $\sigma^{ \pm}$and work in conformal gauge: $g_{+-}=-e^{2 \rho} / 2, g_{++}=g_{--}=0$.

The black hole is formed by collapse of a shock-wave, traveling in the $\sigma^{-}$direction, described by the stress tensorf

$$
\frac{1}{2} \partial_{+} f \partial_{+} f=a e^{-\sigma_{0}^{+}} \delta\left(\sigma^{+}-\sigma_{0}^{+}\right)
$$

\footnotetext{
${ }^{4}$ We choose to write the magnitude of the shock-wave as $a e^{-\sigma_{0}^{+}}$, in order to keep our notation " $a "$ consistent with the one of Ref.[1].
} 
The solution of the MCDG classical equations of motion, taking into account that for $\sigma^{+}<\sigma_{0}^{+}$ we are in the vacuum, gives a black hole background metric with conformal factor

$$
\rho=-\frac{1}{2} \ln \left[1+\Theta\left(\sigma^{+}-\sigma_{0}^{+}\right) \frac{a}{\lambda} e^{\lambda \sigma^{-}}\left(e^{\lambda\left(\sigma_{0}^{+}-\sigma^{+}\right)}-1\right)\right] .
$$

The next step in the conventional semiclassical analysis of this black hole, consists in using the quantum relations (2) and (5) to derive the flux of matter energy across $\mathcal{I}_{R}^{+}$, which is given by the value of $T_{--}$on $\mathcal{I}_{R}^{+}$. In conformal gauge (21) and (5) take the form

$$
\begin{gathered}
T_{+-}=-\frac{N}{12 \pi} \partial_{+} \partial_{-} \rho \\
\partial_{\mp} T_{ \pm \pm}+\partial_{ \pm} T_{+-}-2 T_{+-} \partial_{ \pm} \rho=0,
\end{gathered}
$$

and these lead to

$$
T_{ \pm \pm}=\frac{N}{12 \pi}\left[\partial_{ \pm}^{2} \rho-\left(\partial_{ \pm} \rho\right)^{2}+t_{ \pm}\left(\sigma^{ \pm}\right)\right]
$$

The functions of integration $t_{ \pm}$are to be determined by imposing physical boundary conditions, which for our collapsing shock-wave consist [1] in requiring that $T_{\mu \nu}$ vanish on $\mathcal{I}_{L}^{-}$(i.e. $\left.\sigma^{+}=-\infty\right)$, and that there be no incoming radiation along $\mathcal{I}_{R}^{-}\left(i . e . \sigma^{-}=-\infty\right)$ except for the classical shock-wave at $\sigma^{+}=\sigma_{0}^{+}$; this implies that $t_{ \pm}=0$. Substituting $t_{ \pm}=0$ and (18) in (19) and (21) one easily derives that on $\mathcal{I}_{R}^{+}\left(\right.$i.e. $\left.\sigma^{+} \rightarrow \infty\right)$

$$
\begin{aligned}
& {\left[T_{++}\right]_{\mathcal{I}_{R}^{+}}=0, \quad\left[T_{+-}\right]_{\mathcal{I}_{R}^{+}}=0,} \\
& {\left[T_{--}\right]_{\mathcal{I}_{R}^{+}}=\frac{N a \lambda^{2}}{48 \pi} e^{\lambda \sigma^{-}} \frac{2 \lambda-a e^{\lambda \sigma^{-}}}{\left(\lambda-a e^{\lambda \sigma^{-}}\right)^{2}} .}
\end{aligned}
$$

As clarified in Ref.[1], the physical interpretation of this solutions is clearest in the $y^{ \pm}$ coordinates

$$
y^{+}=\sigma^{+}, \quad y^{-}=-\ln \left(e^{-\lambda \sigma^{-}}-a / \lambda\right) / \lambda,
$$

\footnotetext{
${ }^{5}$ Like in Ref.[1], $\mathcal{I}_{R}^{+}\left(\mathcal{I}_{R}^{-}\right)$is the future (past) null infinity for right-moving light rays, and analogously $\mathcal{I}_{L}^{+}$ $\left(\mathcal{I}_{L}^{-}\right)$is the future (past) null infinity for left-moving light rays.
} 
where the conformal factor takes the form (N.B.: $y_{0}^{+} \equiv \sigma_{0}^{+}$)

$$
\rho=-\frac{1}{2} \ln \left[1+\frac{a}{\lambda} e^{\lambda y^{-}+\lambda \Theta\left(y^{+}-y_{0}^{+}\right)\left(y_{0}^{+}-y^{+}\right)}\right]
$$

and therefore the metric is asymptotically constant on $\mathcal{I}_{R}^{ \pm}$.

Using the fact that $T_{\mu \nu}$ transforms like a tensor under diffeomorphisms, from (22) and (23) one finds [1] that in the $y^{ \pm}$coordinates

$$
\begin{aligned}
{\left[T_{++}\right]_{\mathcal{I}_{R}^{+}} } & =0, \quad\left[T_{+-}\right]_{\mathcal{I}_{R}^{+}}=0, \\
{\left[T_{--}\right]_{\mathcal{I}_{R}^{+}} } & =\frac{N \lambda^{2}}{48 \pi}\left[1-\frac{1}{\left(1+a e^{\lambda y^{-}} / \lambda\right)^{2}}\right] .
\end{aligned}
$$

Eq.(27) gives the flux of energy across $\mathcal{I}_{R}^{+}$. Consistently with the picture of black hole radiation [2], in the far past of $\mathcal{I}_{R}^{+}\left(\right.$i.e. $\left.y^{-} \rightarrow-\infty\right)$ this flux vanishes exponentially, while it approaches a constant value as the horizon $\left(i . e . y^{-} \rightarrow \infty\right)$ is approached.

\subsection{Conformal-Gauge Analysis for Arbitrary $k$}

Let us now generalize the analysis to the case in which the matter energy-momentum tensor satisfies the anomaly relations (9) and (10). Since the anomalies are a quantum effect, nothing changes concerning the black hole background metric, but, instead of (19) and (20), the equations satisfied by the matter energy-momentum tensor in conformal gauge are now given by

$$
\begin{gathered}
T_{+-}=-\frac{N}{12 \pi} k^{2} \partial_{+} \partial_{-} \rho \\
\partial_{\mp} T_{ \pm \pm}+\partial_{ \pm} T_{+-}-2 T_{+-} \partial_{ \pm} \rho=\frac{N}{12 \pi} k(1-k) \partial_{\mp} \partial_{+} \partial_{-} \rho
\end{gathered}
$$

which lead to

$$
T_{ \pm \pm}=\frac{N}{12 \pi}\left[k \partial_{ \pm}^{2} \rho-k^{2}\left(\partial_{ \pm} \rho\right)^{2}+t_{ \pm}\left(\sigma^{ \pm}\right)\right]
$$

Obviously, (28), (29), and (30) reproduce (19), (20), and (21) when $k=1$.

The functions of integration $t_{ \pm}$are to be determined by requiring again that $T_{\mu \nu}$ vanish on $\mathcal{I}_{L}^{-}$, and that there be no incoming radiation along $\mathcal{I}_{R}^{-}$except for the classical shock-wave 
at $\sigma^{+}=\sigma_{0}^{+}$; this leads again to $t_{ \pm}=0$. Then using (28), (30), and the expression of $\rho$ given in (18) we find that in the $\sigma^{ \pm}$coordinates

$$
\begin{aligned}
{\left[T_{++}\right]_{\mathcal{I}_{R}^{+}} } & =0, \quad\left[T_{+-}\right]_{\mathcal{I}_{R}^{+}}=0 \\
{\left[T_{--}\right]_{\mathcal{I}_{R}^{+}} } & =\frac{N a \lambda^{2}}{48 \pi} k e^{\lambda \sigma^{-}} \frac{2 \lambda-k a e^{\lambda \sigma^{-}}}{\left(\lambda-a e^{\lambda \sigma^{-}}\right)^{2}} .
\end{aligned}
$$

In order to get a clear physical interpretation of this result we need to express it in the $y^{ \pm}$coordinates like before. In doing so, we shall take into account the fact that, when $k \neq 1$, $T_{\mu \nu}$ does not transform covariantly under coordinate redefinitions of non-constant Jacobian. For a conformal coordinate redefinition $\sigma^{+} \rightarrow y^{+}=\sigma^{+}, \sigma^{-} \rightarrow y^{-}=f\left(\sigma^{-}\right)$, from (16) one finds that $\Delta_{++}^{(\sigma, y)}=\Delta_{+-}^{(\sigma, y)}=0$, and

$$
\Delta_{--}^{(\sigma, y)}=\frac{N}{24 \pi}(1-k)\left\{\partial_{-}^{2} \ln \left(\frac{d y^{-}}{d \sigma^{-}}\right)-\frac{(1-k)}{2}\left[\partial_{-} \ln \left(\frac{d y^{-}}{d \sigma^{-}}\right)\right]^{2}-2 k\left(\partial_{-} \rho\right) \partial_{-} \ln \left(\frac{d y^{-}}{d \sigma^{-}}\right)\right\},
$$

which generalizes the ordinary 12 $(k=0)$ Schwarzian derivative of the conformal map $\sigma \rightarrow y$, to the case of our $k$-dependent anomalous transformations of the energy momentum tensor.

Since $d \sigma^{-} / d y^{+}=0$, from (15) and (31) it follows that on $\mathcal{I}_{R}^{+}$

$$
\begin{gathered}
{\left[T_{++}^{(y)}\right]_{\mathcal{I}_{R}^{+}}=0, \quad\left[T_{+-}^{(y)}\right]_{\mathcal{I}_{R}^{+}}=0,} \\
{\left[T_{--}^{(y)}\right]_{\mathcal{I}_{R}^{+}}=\left[\left(T_{--}^{(\sigma)}+\Delta_{--}^{(\sigma, y)}\right)\left(d \sigma^{-} / d y^{-}\right)^{2}\right]_{\mathcal{I}_{R}^{+}} .}
\end{gathered}
$$

and with a straightforward calculation we find that

$$
\begin{gathered}
{\left[T_{--}^{(\sigma)}\left(\frac{d \sigma^{-}}{d y^{-}}\right)^{2}\right]_{\mathcal{I}_{R}^{+}}=\frac{N \lambda^{2}}{48 \pi} \frac{2 k a e^{\lambda y^{-}} / \lambda+\left(2 k-k^{2}\right) a^{2} e^{2 \lambda y^{-}} / \lambda^{2}}{\left(1+a e^{\lambda y^{-}} / \lambda\right)^{2}},} \\
{\left[\Delta_{--}^{(\sigma, y)}\left(\frac{d \sigma^{-}}{d y^{-}}\right)^{2}\right]_{\mathcal{I}_{R}^{+}}=\frac{N \lambda^{2}}{48 \pi}\left[1-\frac{1+2 k a e^{\lambda y^{-}} / \lambda+\left(2 k-k^{2}\right) a^{2} e^{2 \lambda y^{-}} / \lambda^{2}}{\left(1+a e^{\lambda y^{-}} / \lambda\right)^{2}}\right] .}
\end{gathered}
$$

Adding these last two results we see that the $k$-dependent terms cancel out, and, obviously, the left-over formula for $T_{--}$on $\mathcal{I}_{R}^{+}$exactly reproduces Eq.(27). We conclude that the black hole radiation observed in the $y^{ \pm}$coordinate system is insensitive to the value of $k$.

Using the covariance of $T_{\mu \nu}$ discussed in Section 2, we can deduce that the coordinate systems which can be obtained from the $y^{ \pm}$coordinate system by a coordinate redefinition of 
Jacobian asymptotically constant on $\mathcal{I}_{R}^{+}$will also observe $k$-independent black hole radiation. Importantly, these are all the coordinate systems with metric asymptotically constant on $\mathcal{I}_{R}^{+}$, which obviously include all observers asymptotically Minkowskian on $\mathcal{I}_{R}^{+}$.

\subsection{Light-Cone-Gauge Analysis for Arbitrary $k$}

We now want to show that also for the light-cone-gauge observers, which we define as those with $g_{--}=0$ and $g_{+-}=-1 / 2$, the black hole radiation is $k$-independent. Let us start by observing that the anomaly relations (12) and (13) imply that

$$
\hat{\nabla}_{\mu}\left(\hat{g}^{\mu \nu} T_{\nu \alpha}\right)-\frac{1}{2} \partial_{\alpha}\left(\hat{g}^{\mu \nu} T_{\mu \nu}\right)=-\frac{N}{48 \pi} \partial_{\alpha} R(\hat{g})
$$

which does not depend explicitly on $k$; it depends on $k$ only implicitly, through the $k$ dependence of $\hat{g}_{\mu \nu}$. This relation is particularly useful in light-cone gauge, where the metric $g_{\mu \nu}$ has constant determinant, and therefore the $k$-dependence of $\hat{g}_{\mu \nu}$ is trivial.

Using (38) and (9), one finds that in light-cone gauge the matter energy-momentum tensor satisfies the relations

$$
\begin{gathered}
T_{+-}+g_{++} T_{--}=-\frac{N}{24 \pi} k \partial_{-}^{2} g_{++} \\
\partial_{-} T_{++}+2 \partial_{-}\left(g_{++} T_{+-}\right)-g_{++} \partial_{+} T_{--}=\frac{N}{24 \pi} \partial_{+} \partial_{-}^{2} g_{++} \\
\partial_{+} T_{--}+2 \partial_{-}\left(g_{++} T_{--}\right)-g_{++} \partial_{-} T_{--}=\frac{N}{24 \pi} \partial_{-}^{3} g_{++}
\end{gathered}
$$

Most importantly, the differential equation (41) involves only $T_{--}$and is $k$-independent; therefore, with $k$-independent boundary conditions, it leads to $k$-independent $T_{--}$. The general solution of (41) has the form

$$
T_{--}=\frac{N}{24 \pi}\left(\partial_{-} F\left(\xi^{+}, \xi^{-}\right)\right)^{2}\left[g_{++}\left(\xi^{+}, \xi^{-}\right)\left\{F\left(\xi^{+}, \xi^{-}\right), \xi^{-}\right\}+\frac{1}{2} \partial_{-} g_{++}^{2}\left(\xi^{+}, \xi^{-}\right)+t_{-}^{l c}\left(F\left(\xi^{+}, \xi^{-}\right)\right)\right]
$$

where $\xi^{+}$and $\xi^{-}$are light-cone coordinates, $F$ is such that

$$
g_{++}=-\frac{\partial_{+} F}{\partial_{-} F}
$$


$\{$,$\} denotes the ordinary Schwarzian derivative, and t_{-}^{l c}$, which is a function of $\xi^{+}$and $\xi^{-}$ only through $F$, is to be fixed by imposing physical boundary conditions.

It is easy to verify explicitly that, for light-cone-gauge observers, the black hole radiation is insensitive to the value of $k$. In light-cone gauge the black hole background metric that we have been considering can be described by (N.B.: the shock-wave is at $\xi^{+}=\xi_{0}^{+}$)

$$
g_{++}=1+\Theta\left(\xi^{+}-\xi_{0}^{+}\right)\left[a e^{\lambda\left(\xi^{-}-\xi^{+}+\xi_{0}^{+}\right)} / \lambda-1\right]
$$

which corresponds to

$$
F=-\frac{1}{\lambda} \ln \left[e^{\lambda\left(\xi^{+}-\xi^{-}-\xi_{0}^{+}\right)}-a / \lambda\right]+\Theta\left(\xi^{+}-\xi_{0}^{+}\right)\left(\xi^{+}-\xi_{0}^{+}\right)
$$

We observe that this $F$ also has a geometrical interpretation; in fact, the $\xi^{ \pm}$coordinates that we are using in light-cone gauge and the $y^{ \pm}$coordinates that we used in the preceding subsections are related by

$$
y^{+}=\xi^{+}, \quad y^{-}=F\left(\xi^{+}, \xi^{-}\right)
$$

The physical boundary conditions needed to fix $t_{-}^{l c}\left(\xi^{+}, \xi^{-}\right)$are again $[$provided by the requirement that $T_{\mu \nu}$ vanish on $\mathcal{I}_{L}^{-}$and that there be no incoming radiation along $\mathcal{I}_{R}^{-}$except for the classical shock-wave at $\xi^{+}=\xi_{0}^{+}$. This leads to

$$
t_{-}^{l c}=\frac{\lambda^{2}}{2}\left[1-\frac{1}{\left(1+a e^{\lambda F\left(\xi^{+}, \xi^{-}\right)} / \lambda\right)^{2}}\right] .
$$

Eqs.(42), (44), (45), and (47) completely determine $T_{--}$, and in particular on $\mathcal{I}_{R}^{+}$one finds that

$$
\left[T_{--}\right]_{\mathcal{I}_{R}^{+}}=\frac{N \lambda^{2}}{48 \pi}\left[1-\frac{1}{\left(1+a e^{\lambda \xi^{-}} / \lambda\right)^{2}}\right]
$$

${ }^{6}$ Since the $\sigma^{ \pm}$coordinates are asymptotically Minkowskian on $\mathcal{I}_{L}^{-}$and $\mathcal{I}_{R}^{-}$(see Eq.(18)), there is a coordinate redefinition of Jacobian asymptotically constant on $\mathcal{I}_{L}^{-}$and $\mathcal{I}_{R}^{-}$which connects the $\sigma^{ \pm}$coordinate system and any given light-cone gauge coordinate system. Therefore, in order to obtain the corresponding boundary conditions in a given light-cone gauge coordinate system, we can transform covariantly the boundary conditions for $T_{\mu \nu}$ imposed on $\mathcal{I}_{L}^{-}$and $\mathcal{I}_{R}^{-}$in the $\sigma^{ \pm}$coordinate system. 
which, as expected, indicates that the black hole radiation observed in the $\xi^{ \pm}$coordinates is insensitive to the value of $k$. Since any two light-cone-gauge observers are connected by a diffeomorphism of unit Jacobian, which is a symmetry of the theory for any $k$, the $k$ independence of the black hole radiation observed in the $\xi^{ \pm}$coordinates also applies to any other light-cone-gauge observer.

Note that (48) is identical to (27). This is due to the fact that, as shown by (44), also in the $\xi^{ \pm}$coordinates the metric is asymptotically constant on $\mathcal{I}_{R}^{+}$, and, as shown by (46), the map between $\xi^{+}, \xi^{-}$and $y^{+}, y^{-}$is the identity on $\mathcal{I}_{R}^{+}$.

For completeness we also notice that, having solved for $T_{--}$and fixed the above mentioned boundary conditions, one can use (39) and (40) to derive $T_{++}$and $T_{+-}$, and in particular on $\mathcal{I}_{R}^{+}$one finds again that

$$
\left[T_{++}\right]_{\mathcal{I}_{R}^{+}}=0, \quad\left[T_{+-}\right]_{\mathcal{I}_{R}^{+}}=0
$$

\section{Conclusion}

To summarize, in our semiclassical analysis of black hole radiation in matter-coupled dilaton gravity, we have considered a one-parameter $k$-family of measures for the path integral quantization of the matter fields. We have derived several symmetry properties of these measures, including a formula for the non-covariant transformation of the matter energymomentum tensor under coordinate redefinitions, and observed that the Weyl anomaly is proportional to the parameter $k$. We have found that all these quantizations of the matter fields are consistent with the phenomena of black hole radiation, and that the radiation seen by all observers whose metric is asymptotically constant on $\mathcal{I}_{R}^{+}$, which are the observers ordinarily used in the description of black hole radiation, is insensitive to the value of $k$. We have verified explicitly this $k$-independence for two such observers, one in conformal gauge and the other in light-cone gauge, and used the covariant conservation of the matter energymomentum tensor under coordinate redefinitions of constant Jacobian to deduce its validity for any other such observer. 
Our results should also clarify the relation between anomalies and black hole radiation in $1+1$ dimensions. The usual claim that the black hole radiation is a consequence of the Weyl anomaly, should be understood as strongly dependent on the assumption that the matter energy-momentum tensor be covariantly conserved at the quantum level. In general, the presence of any (Weyl and/or diffeomorphism) anomaly is sufficient to support black hole radiation. The insensitivity of black-hole radiation to the parameter characterizing the measure must be understood as a feature of the particular (bosonic) theory that we considered; in fact, based on the experience with the chiral Schwinger model[14 (where a one-parameter "a"-family of chiral symmetry breaking measures has been investigated, and the mass emergent in that theory does depend on $a$ ), one can expect that parameters characterizing the measure have a non-trivial physical role in more general theories (particularly when gravity is coupled to chiral matter [11, 15]).

Interestingly, in our light-cone-gauge analysis a key role was played by the relation (38), which in every gauge depends only implicitly on $k$ and in light-cone gauge is completely $k$-independent. This relation generalizes the one $(k=0)$ encountered in Ref. [10] to the case of the $k$-dependent anomalies (91),(10). The results found in the present paper agree with the expectation [10] that this relation encodes some essential feature of the theory.

We also observe that the singularity of the limit $k \rightarrow 0$ in Eq.(7) was not encountered in any of the results which have followed. Further investigation of the possible consequences of this singularity would be interesting. It is plausible that it may surface as a non-analyticity to be handled in the higher orders of the semiclassical approximation, but it may also turn out to be simply an accidental result of the type of parametrization that we have chosen.

We thank E. Keski-Vakkuri for suggesting that the results of Ref. [10] might be important for the understanding of the relation between Weyl anomaly and black hole radiation, and L. Griguolo and R. Jackiw for very useful comments. 


\section{Note Added}

Upon completion of our manuscript, L. Griguolo brought to our attention Ref.[16], in which 1+1-dimensional black hole radiation is analyzed semiclassically in conformal gauge assuming that the matter energy-momentum tensor be traceless, but not covariantly conserved, i.e. the special case $k=0$ in the one-parameter $k$-family of quantizations that we considered here. 


\section{References}

[1] For a recent review, see A. Strominger, Les Houches Lectures on Black Holes, hepth/9501071.

[2] S.W. Hawking, Comm. Math. Phys. 43 (1975) 199.

[3] S. M. Christensen and S. A. Fulling, Phys. Rev. D15 (1977) 2088.

[4] M. Bos, Phys. Rev. D34 (1986) 3750.

[5] A.M. Polyakov, Phys. Lett. 103B (1981) 207; A.M. Polyakov, Mod. Phys. Lett. A2 (1987) 893; V. Knizhnik, A.M. Polyakov and A. Zamolodchikov, Mod. Phys. Lett. A3 (1988) 819; J. Distler and H. Kawai, Nucl. Phys. B321 (1988) 171; F. David, Mod. Phys. Lett. A3 (1988) 1651; A. Bilal and I.I. Kogan, LPTENS-95-14/OUTP-95-11P (1995).

[6] L. Bonora, P. Pasti, and M. Bregola, Class. Quant. Grav. 3 (1986) 635.

[7] M. Hatsuda, P. van Nieuwenhuizen, W. Troost and A. van Proeyen, Nucl. Phys. B355 (1990) 166.

[8] D.R. Karakhanian, R.P. Manvelian, R.L. Mkrtchian, Phys. Lett. B329 (1994) 185.

[9] R. Jackiw, MIT-CTP-2377/hep-th-9501016 (1995).

[10] G. Amelino-Camelia, D. Bak, and D. Seminara, Physics Letters B354 (1995) 213.

[11] G. Amelino-Camelia, L. Griguolo, and D. Seminara, in preparation.

[12] See, for example, C. Itzykson and J.-M. Drouffe, Statistical Field Theory, (Cambridge University Press, 1989).

[13] C.G. Callan, S.B. Giddings, J.A. Harvey, and A. Strominger, Phys. Rev. D45 (1992) 1005.

[14] R. Jackiw and R. Rajaraman, Phys. Rev. Lett. 54 (1985) 1219; A. Bassetto, L. Griguolo, and P. Zanca, Phys. Rev. D50 (1994) 1077; for a review see R. Jackiw in Quantum Mechanics of Fundamental Systems 1, C. Teitelboim ed. (Plenum, New York, 1988).

[15] L. Griguolo, Class. Quant. Grav. 12, 1165 (1995).

[16] J. Navarro-Salas, M. Navarro, and C. F. Talavera, Phys. Lett. B356 (1995) 217. 